\title{
Tabularia
}

TABULARIA Sources écrites des mondes normands médiévaux Guillaume de Volpiano : Fécamp et l'histoire normande | 2002

\section{Guillaume de Volpiano en Normandie : état des questions}

William of Volpiano in Normandy: current position

\section{Véronique Gazeau}

\section{OpenEdition}

Journals

\section{Édition électronique}

URL : http://journals.openedition.org/tabularia/1756

DOI : 10.4000/tabularia. 1756

ISSN : 1630-7364

Éditeur :

CRAHAM - Centre Michel de Boüard, Presses universitaires de Caen

\section{Référence électronique}

Véronique Gazeau, « Guillaume de Volpiano en Normandie : état des questions », Tabularia [En ligne], Guillaume de Volpiano : Fécamp et l'histoire normande, mis en ligne le 22 juillet 2002, consulté le 30 avril 2019. URL : http://journals.openedition.org/tabularia/1756; DOI : 10.4000/tabularia.1756 


\title{
Guillaume de Volpiano en Normandie : état des questions
}

\section{William of Volpiano in Normandy : current position}

\author{
Véronique GAZEAU \\ CRAHM-UMR 6577, \\ Université de Caen Basse-Normandie
}

Résumé:

L'appel de Richard II au réformateur italo-bourguignon Guillaume de Volpiano en 1001 marque une étape majeure dans le relèvement du monachisme dans la principauté normande. Fécamp devient l'abbaye ducale d'où partent les initiatives des disciples de Guillaume vers Jumièges, Bernay, Le Mont-Saint-Michel, et au-delà Troarn, Saint-Taurin d'Évreux, Conches et Sées. L'influence de Guillaume de Volpiano est particulièrement visible dans le domaine liturgique et architectural. Mais le prestige de Fécamp ne doit pas éclipser le rôle d'autres réformateurs issus de régions extérieures à la Normandie, et qui ont eu leur part, avant l'arrivée de Guillaume et après sa mort (1031), dans le renouveau monastique du duché.

Mots-clés: Monachisme, Guillaume de Volpiano, Fécamp, Richard II.

\section{Abstract:}

Richard II's appeal to William of Volpiano, an Italian-Burgundian reformer in 1001, must be regarded as an important stage in the revival of monasticism in the Norman principality. Fécamp becomes the ducal abbey, from where William's disciples initiate reforms at Jumièges, Bernay, le Mont-Saint-Michel and the abbeys of Troarn, Saint-Taurin d'Evreux, Conches, and Sées. William of Volpiano's influence is evident in liturgical and architectural matters. One cannot forget that other foreign reformers came to Normandy before William of Volpiano and after his death (1031) who had a part in the settling of monasticism in the duchy.

Keywords: Monasticism, William of Volpiano, Fécamp, Richard II.

Le prestige de l'abbaye de Fécamp fut incomparablement supérieur à celui des trente-trois autres abbayes bénédictines que compte la Normandie. Selon la formule de Raoul Glaber, «l'heureuse prospérité de Fécamp éclipsait tous les autres établissements »'. En effet, et en premier lieu, l'abbaye fut le lieu de sépulture des

1. Vie de saint Guillaume abbé de Dijon, chap. VII, GAZEAU, Véronique (trad.), La Normandie vers l'an mil, François de Beaurepaire et Jean-Pierre Chaline (coord.), Rouen, Société de l'Histoire de Normandie, 2000, p. 133. 
princes normands Richard Ier et Richard II et de plusieurs membres de la famille ducale $^{2}$. En deuxième lieu, l'étude prosopographique démontre que le choix des successeurs de Guillaume de Volpiano ne relève pas seulement des compétences religieuses. Jean de Ravenne est un disciple de Guillaume, le choix semble avoir été effectué de concert avec le duc Robert le Magnifique ${ }^{3}$. Guillaume de Rots, le troisième abbé, a été moine de Saint-Etienne de Caen, la fondation de Guillaume le Conquérant, sous l'abbatiat de Guillaume Bonne Âme ${ }^{4}$. En 1079, les deux hommes sont promus, le premier à Fécamp, le second au siège métropolitain de Rouen. Le quatrième abbé de Fécamp, Roger de Bayeux, est un disciple de Guillaume de Rots et doit sa désignation au duc-roi Henri $1^{\text {er }}$ Beauclerc. L'abbé Henri de Sully est le propre neveu du futur roi d'Angleterre, Etienne de Blois ${ }^{5}$. En troisième lieu, les successeurs de Guillaume de Volpiano figurent très souvent les premiers souscripteurs ou témoins dans les listes des abbés au bas des diplômes ducaux. Ils sont parfois seuls présents au bas des mêmes diplômes. Leur présence est d'autant plus remarquable que sont rares les souscriptions abbatiales dans la Normandie ducale. Enfin, des événements de la plus haute importance se déroulent à Fécamp aux XI ${ }^{e}$-XII ${ }^{e}$ siècles. À Pâques 1075, Guillaume le Conquérant se rend à l'abbaye alors que sa fille Cécile prononce ses vœux monastiques. Il y revient en $1083^{6}$. Le 3 mars 1162, le duc-roi Henri II assiste à la translation des corps des ducs Richard I Ir et Richard II, au-dessus de l'autel majeur? ${ }^{7}$. Si certains historiens - Colette Beaune et Mathieu Arnoux - peuvent considérer que les ducs sont moins présents à Fécamp après la fondation de Saint-Etienne de Caen ${ }^{8}$, les papes ont accordé à l'abbaye une attention toute particulière. Alexandre III, en 1174, remet à l'abbé Henri les insignes pontificaux, la mitre, l'anneau et les sandales, faveur confirmée par Lucius III en $1182^{9}$. En 1197, Célestin III autorise l'abbé Raoul à porter la tunique et la dalmatique ${ }^{10}$. Aucun autre abbé en Normandie n'a reçu de tels privilèges, octroyés ici prématurément, puisque c'est généralement

2. DUdON DE SAINT-QUENTIN, De moribus et actis primorum Normanniae ducum, éd. Jules LAIR, M.S.A.N., 23, 1865, p. 297-299. VAN HoUTS, Elisabeth, Gesta Normannorum ducum, Oxford, 1992-1995, II, p. 38-40. ORDÉRIC VITAL, Histoire ecclésiastique, éd. Marjorie CHIBNALL, II, p. 118.

3. Chronique de l'abbaye de Saint Bénigne, éd. Louis-Emile BOUGAUD et Joseph GARNIER, Dijon, 1875 , p. 157.

4. ORDERIC VITAL, Histoire ecclésiastique, II, p. 150.

5. ORDERIC VITAL, Histoire ecclésiastique, VI, p. 536.

6. ORderic VITAL, Histoire ecclésiastique, III, p. 8. BATES, David, Regesta Regum Anglo-Normannorum. The Acta of William I (1066-1087), Oxford, 1998, $\mathrm{n}^{\circ} 230$.

7. Annales du Mont-Saint-Michel, Léopold DelisLe (éd.), II, p. 228.

8. BeaUne, Colette, "Les ducs, le roi et le Saint Sang », in Saint-Denis et la royauté. Etudes offertes à Bernard Guenée, Paris, 1999, p. 711-732. ARNoux, Mathieu, «A. D. MI : Willelmus abbas Fiscannensis efficitur année du Seigneur 1001 : Guillaume devient abbé de Fécamp », De l'histoire à la légende, La broderie du Précieux-Sang, Catalogue des Musées municipaux de Fécamp, Fécamp, 2001, p. 8.

9. RAMACKers, Johannes, Papsturkunden in Franfreich, N. F. 2 : Normandie, Göttingen, 1937, $\mathrm{n}^{\circ} 212$.

10. Ibid., $\mathrm{n}^{\circ} 339$. 
sous le pontificat d'Innocent III, donc à partir de 1198, que les pontificalia commencent à être exceptionnellement donnés.

À n'en pas douter, l'abbatiat de Guillaume de Volpiano appelé par le duc Richard II à réformer Fécamp a contribué à donner éclat et renommée à l'abbaye ducale. L'historiographie s'est efforcée d'accorder à Guillaume de Volpiano une sorte de paternité dans la réforme du monachisme normand. Au vide du $\mathrm{X}^{\mathrm{e}}$ siècle dû aux exactions des Vikings, vide qui devait concerner tous les aspects de la vie de la Normandie, il convenait d'opposer le renouveau, dans le domaine monastique, voulu ex nihilo par les ducs et Guillaume de Volpiano. Lors de la célébration du treizième Centenaire de l'abbaye de Fécamp en 1958, René Herval concluait sa communication intitulée «Un moine de l'an mille : Guillaume de Volpiano, premier abbé de Fécamp (962-1031) », ainsi : «À la mort de Guillaume de Volpiano, la page de l'Histoire de l'an mille était tournée... De cette résurrection, le premier abbé de Fécamp et ses disciples avaient été les précurseurs et les meilleurs annonciateurs. Cela suffit à leur gloire et justifie largement la haute admiration que la Normandie garde à leur souvenir $»^{11}$. Depuis 1958, les historiens dans le sillage des travaux menés par Jean-François Lemarignier, Lucien Musset, David Douglas, Marjorie Chibnall ${ }^{12}$, pour ne citer que ceux-là, et surtout depuis une bonne décennie, ont commencé de revisiter les sources de l'histoire du duché de Normandie. Entre temps, en 1973, paraissait la thèse de Neithard Bulst, consacrée aux réformes de Guillaume de Dijon, thèse qui demeure un ouvrage de référence ${ }^{13}$. En précurseur, Neithard Bulst mettait en place des chronologies, dressait une prosopographie des abbés qui gravitaient dans le sillage de Guillaume, pour finalement limiter la portée des réformes de ce dernier en Normandie.

Que peut-on dire aujourd'hui de l'œuvre de l'abbé Guillaume de Volpiano? Quelle fut son action à Fécamp et en Normandie, de 1001, date de son arrivée dans le duché, à 1031, date de sa mort à Fécamp ? Et quelle fut la portée de son œuvre en Normandie, au-delà de sa mort? Evoquons quelques pistes de recherches et quelques éléments de réponses en dressant un état de la recherche en cours, aujourd'hui en France mais aussi Outre-Rhin, Outre-Manche et aux ÉtatsUnis.

11. HerVAL, René, «Un moine de l'an mille : Guillaume de Volpiano, premier abbé de Fécamp (9621031) ", in L'abbaye bénédictine de Fécamp, ouvrage scientifique du XIII centenaire, 658-1958, Fécamp, 1958-1960, I, p. 44.

12. LEMARIGNIER, Jean-François, Etude sur le privilège d'exemption et de juridiction ecclésiastique des abbayes normandes depuis les origines jusqu'en 1140, Paris, 1937. MUSSET, Lucien, "La contribution de Fécamp à la reconquête monastique de la Basse-Normandie ", dans L'abbaye bénédictine de Fécamp. Ouvrage scientifique du XIII centenaire, 658-1958, Fécamp, 1959, p. 57-68 et p. 341343. Douglas, David, "La première charte ducale pour Fécamp ", L'abbaye bénédictine de Fécamp, ouvrage scientifique du XIII centenaire, 658-1958, Fécamp, 1958-1960, I, p. 44-56 et 327339. CHIBNALL, Marjorie, « Fécamp and England ", L'abbaye bénédictine de Fécamp, ouvrage scientifique du XIII centenaire, 658-1958, Fécamp, 1958-1960, I, p. 126-135 et 367-378.

13. BULST, Neithard, Untersuchungen zu den Klosterreformen Wilhelms von Dijon (962-1031), Bonn, Ludwig Röhrscheid Verlag, 1973, 330 p. 
Raoul Glaber, un moine bourguignon de ses disciples, rédigea en quatorze chapitres la vita de son maître, quelque temps après $1031^{14}$. Né en juin ou juillet 962 dans la citadelle de San Giulio d'une lignée aristocratique alémanique, oblat à Lucedio au diocèse de Verceil, Guillaume ne devint pas moine de Cluny par hasard. Mais on ne s'attardera pas à ces moments-là de sa vie. Il réforme l'abbaye de Saint-Bénigne de Dijon à partir de 989 et se voit confier dans les années suivantes plusieurs monastères en Bourgogne, Lorraine et Ile-de-France. En 1000-1001 il fonde Fruttuaria en Italie du Nord.

Et c'est en 1001 - il y a mille ans aujourd'hui - que le duc Richard II de Normandie appelle à Fécamp Guillaume de Volpiano encore appelé Guillaume de Dijon, précédé par sa réputation d'abbé réformateur. Raoul Glaber présente au chapitre VII de la Vita l'action de Guillaume à Fécamp et insiste sur les largesses effectuées en faveur de l'abbaye sitôt l'arrivée du nouvel abbé ${ }^{15}$. Celles-ci, confirmées par les sources diplomatiques, sont constituées en 1006 de domaines sis dans le comté de Caux, à Granville, Arques, à Ecretteville, à Harfleur, à Rouen, Pissy, Barentin, au Vaudreuil, à Aizier et à Hennequeville ${ }^{16}$. Entre 1017 et 1025, le duc enrichit encore de ses bienfaits l'abbaye en lui remettant des possessions à nouveau dans le pays de Caux ${ }^{17}$. Enfin, un acte de 1025, confirmant les libéralités de Richard I ${ }^{\text {er }}$ - les importants domaines de Saint-Valéry-en-Caux, Argences et Mondeville -, ajoute une quantité considérable de donations dans tout le duché, notamment dans le diocèse de Bayeux ${ }^{18}$. Les nombreux domaines ruraux, les forêts, les moulins, les marchés, les pêcheries, les vignes et autres revenus produisent des ressources susceptibles d'entretenir la communauté et de nourrir les pauvres, comme le suggère la Vita de Guillaume. L'abbé nomme en 1025 les prêtres de 35 églises rurales. En 1006, l'abbaye est dotée de l'exemption. Quelques années plus tard, elle est placée sous la protection pontificale, tout en restant dans l'orbite ducale $^{19}$. On ignore tout des écoles mentionnées par le biographe de Guillaume qui connaît certainement la grammaire. On ne conserve aucune œuvre de l'abbé de Fécamp. Sans doute créa-t-il une école pour les oblats et une autre pour les clercs. On y apprend à déchiffrer l'Ecriture sainte, à prier et chanter les psaumes. Raoul Glaber insiste sur la décadence qui règne dans le domaine du chant en Normandie et sur l'influence qu'a eue Guillaume de Volpiano dans le détail de l'organisation des chants de l'office. Il faut très vraisemblablement considérer qu'il a lui-même composé des antiennes et des répons, que l'on ne rencontre que dans les manuscrits des monastères qu'il a lui-même réformés ${ }^{20}$. La Bibliothèque municipale de

14. RaOUl Glaber, "Vita domni Willelmi abbatis ", Rodulfus Glaber opera, Neithard Bulst et John FRANCE (éd. et trad. anglaise), Oxford (Oxford Medieval Texts), 1999, p. 254-299.

15. Vie de saint Guillaume abbé de Dijon..., p. 132-133.

16. Fauroux, Marie, Recueil des actes des ducs de Normandie, Caen, 1961, $\mathrm{n}^{\circ} 9$.

17. Fauroux, Recueil..., n ${ }^{\circ} 31$.

18. Fauroux, Recueil..., n $\mathrm{n}^{\circ} 34$.

19. Sur la question de l'exemption à Fécamp, LEMARIGNIER, Jean-François, Etude sur le privilège...

20. RAOUl Glaber, «Vita domni Willelmi », p. 286-288. 
Rouen conserve un antiphonaire-hymnaire du XIII siècle, précédé d'un traité de musique et d'un tonaire ${ }^{21}$. Or ce tonaire en usage à Fécamp s'apparente à celui de Saint-Bénigne de Dijon, connu par un manuscrit conservé à la Faculté de Médecine de Montpellier et attribué à Guillaume de Volpiano ${ }^{22}$.

Il ne semble pas que les bâtiments claustraux mentionnés par la Vita aient abrité un atelier d'enluminure par exemple. Les moines qui accompagnent Guillaume de Volpiano ont trouvé une Règle des chanoines dans la bibliothèque, un manuscrit de tradition carolingienne, de style franco-saxon avec des lettres ornées $^{23}$. Il faut attendre l'abbatiat de son successeur, Jean (1028-1078), pour que de nouveaux décors se fassent jour ${ }^{24}$. Si, comme le laisse entendre son biographe, Guillaume n'a fait que construire les bâtiments abbatiaux, effectivement, en 990 l'église avait été relevée. Le Libellus de revelatione, edificatione et auctoritate Fiscannensis monasterii précise que des bâtiments claustraux, des ateliers et des maisons ont été édifiés par Richard II, avant l'arrivée de Guillaume ${ }^{25}$. Dudon de SaintQuentin décrit un vaste massif occidental à trois tours ${ }^{26}$.

Guillaume de Volpiano avait introduit à Dijon les coutumes de Cluny qu'il adapta. Fécamp emprunte beaucoup aux coutumes clunisiennes, mais diverge sur plusieurs points.

Si l'on examine l'influence de Guillaume en Normandie, son rôle dépasse largement le cadre du monastère ducal. De son vivant, il s'est efforcé de réformer d'autres établissements au moyen du cumul, comme l'a montré Neithard Bulst ${ }^{27}$ :

Il est non seulement abbé de Fécamp, mais aussi depuis 1025 le premier abbé de Bernay, la fondation de l'épouse de Richard II, Judith, vers 1008-101728. A Jumièges, il a exercé l'abbatiat de 1015 à $1017^{29}$. En réalité Guillaume résida assez peu en Normandie pendant trente ans. Il s'efforce de nommer ses disciples aux postes d'abbé ou de prieur, derrière lesquels il s'efface :

21. Bibl. mun. Rouen, A $190, \mathrm{f}^{\circ} 2-28 \mathrm{v}^{\circ}$.

22. Bibliothèque de la Faculté de Médecine de Montpellier, H 159. Huglo, Michel, «Le tonaire de Saint-Bénigne de Dijon ", Annales musicologiques, 1956, IV, p. 7-18. Un tonaire comprend les incipit des 3000 antiennes de l'office et/ou ceux des 250 antiennes de la messe, classés suivant les huit tons du chant grégorien et, pour chaque ton, il donne la terminaison psalmodique capable de procurer le meilleur enchaînement musical de cette cadence finale à la reprise des antiennes citées. Une antienne est un refrain repris par le choeur entre chaque verset d'un psaume ou chanté seulement avant et après. Un antiphonaire est un recueil des antiennes et des répons ; un bréviaire comprend le lectionnaire et l'antiphonaire.

23. BnF, ms lat. 1535.

24. AVRIL, François, « La décoration des manuscrits dans les abbayes bénédictines de Normandie aux $\mathrm{XI}^{\mathrm{e}}$ et XII ${ }^{\mathrm{e}}$ siècles ", Positions de thèses de l'Ecole des chartes, 1963, p. 24-25.

25. Patrologie latine, t. CLI, col. 719-720.

26. De moribus et actis primorum Normanniae ducum, p. 291. BAYLÉ, Maylis, L'architecture normande au Moyen Age, 1, Regards sur l'art de bâtir, Caen, 1997, II, p. 153.

27. Bulst, Neithard, Untersuchungen..., p. 131 et suiv.

28. FAUrouX, Recueil, n ${ }^{\circ} 35$.

29. BULST, Neithard, Untersuchungen..., p. 165. 
- à Fécamp : à la demande du duc Robert, il cède la place à Jean de Ravenne en $1028^{30}$;

- à Bernay : à une date inconnue (avant 1027), il est aidé ou remplacé par un custos ou gardien, Thierry, ancien moine de Dijon et prieur de Fécamp ${ }^{31}$;

- à Jumièges : en 1017, il place Thierry qui cumule les abbatiats de Jumièges et de Bernay. Les Annales de Jumièges indiquent explicitement qu'il introduit à Jumièges les coutumes dijonnaises ${ }^{32}$.

- au Mont-Saint-Michel : le même Thierry succède à l'Italien Suppo, en $1023^{33}$.

Que reste-t-il de l'œuvre de Guillaume de Volpiano après 1031, en Normandie? La réponse s'appuie sur quelques exemples.

Premièrement, le monachisme qui prend son essor dans les années 1030 avec la multiplication des fondations aristocratiques, relayant l'action des ducs, présente des similitudes avec le monachisme de Guillaume de Volpiano. Les nouvelles abbayes que sont Préaux, Cormeilles, Grestain et d'autres ont à leur tête un supérieur, appelé parfois custos, qui ne porte pas le titre abbatial, le temps que s'affermisse la jeune maison et qu'elle se détache de la maison-mère dans l'orbite de laquelle elle a été créée ${ }^{34}$. Peut-être faut-il voir en cette pratique une influence de Guillaume qui, on l'a vu, avait instauré la pratique du gardiennage à Bernay. Jean de Ravenne cumule de 1052 à 1054 les abbatiats de Fécamp et de Saint-Bénigne de Dijon ${ }^{35}$. Rainier, deuxième abbé de La Trinité-du-Mont de Rouen a très vraisemblablement exercé quelque temps la responsabilité de premier abbé du Tréport fondé en 1059, exemple de cumul à la manière de Guillaume de Volpiano ${ }^{36}$. Le cumul ne doit pas être interprété comme la volonté d'imposer un monachisme autoritaire, mais comme une pratique de gouvernement dans une période délicate.

Deuxièmement, deux abbayes relèvent d'un statut particulier en Normandie. Il s'agit de Bernay et de Saint-Taurin d'Evreux, cette dernière restaurée par Richard $\mathrm{I}^{\mathrm{er}}$ dans la deuxième moitié du $\mathrm{X}^{\mathrm{e}}$ siècle et confiée à Fécamp par Robert le Magnifique en 1034. Dans le giron de Fécamp, elles sont contraintes de choisir un abbé fécampois et de suivre les coutumes monastiques de leur abbaye-mère. Après deux custos, Bernay a un véritable abbé dans les années 1060, Vital ${ }^{37}$.

30. Chronique de l'abbaye de Saint-Bénigne de Dijon, p. 157.

31. Robert DE TORIGNI, De immutatione ordinis monachorum, éd. Léopold DeLISLE, Rouen, 18721873, II, p. 194.

32. Annales de l'abbaye de Saint-Pierre de Jumièges, éd. Dom Jean LAPORTE, 1954, p. 85.

33. De abbatibus Montis sancti Michaelis in Periculo maris, éd. Philippe LaBBE, Nova bibliotheca manuscriptorum, Paris, 1657, I, p. 351.

34. Préaux : Inventio et miracula sancti Vulfranni, Jean LAPORTE (éd.), Société de l'Histoire de Normandie, Mélanges, $14^{e}$ série, Rouen-Paris, 1938, p. 52. Cormeilles : ORdERIC VITAL, Histoire ecclésiastique, II, p. 90. Grestain : Neustria Pia, Rouen, 1663, p. 530.

35. Gallia christiana, t. XI, col. 207 A.

36. Robert De TORIGNi, De immutatione..., II, p. 201. Delisle, Léopold, Rouleaux des morts du IXe au $X V^{e}$ siècle, Paris, 1866, p. 137.

37. ORDERIC VITAL, Histoire ecclésiastique, II, p. 134. 
Troisièmement, plusieurs abbayes vont être en quelque sorte placées dans la mouvance fécampoise par l'entremise d'abbés venus de Fécamp. Après la mort de Guillaume de Volpiano, le Mont-Saint-Michel a encore des abbés fécampois jusqu'au milieu du $\mathrm{XI}^{\mathrm{e}}$ siècle $^{38}$. Conches et Troarn, fondations aristocratiques de 1035 et 1059, choisissent leurs premiers abbés parmi les moines de Fécamp ${ }^{39}$. Le premier abbé de Saint-Martin de Sées vers 1057 est sorti de Troarn, en apportant les coutumes de Fécamp ${ }^{40}$.

Quatrièmement, dans le domaine liturgique, les travaux de Dom Hesbert, de Michel Huglo, de Dom Le Roux et plus récemment d'Olivier Diard, montrent que pour l'office, Guillaume de Volpiano a imposé le cursus bénédictin dans quelques monastères rénovés, c'est-à-dire 12 leçons et 12 répons répartis sur les trois nocturnes pour les fêtes solennelles ${ }^{41}$. Concernant le répertoire liturgique, son influence se vérifie par la présence de pièces originales probablement composées par lui, l'adaptation d'autres pièces par des variantes musicales et littéraires, ce que Raoul Glaber appelle des "corrections ", et leur ordre dans certaines solennités ${ }^{42}$. Son influence apparait dans les quatre dimanches de l'Avent avec un choix de répons et d'antiennes présentés dans le même ordre dans les antiphonaires et bréviaires manuscrits de Fécamp, de Jumièges, du Mont-Saint-Michel, de Saint-Martin de Troarn et indirectement de Conches $^{43}$. Le choix des répons pour les Jeudi, Vendredi et Samedi Saints permet de relever un cursus original dans les abbayes de Fécamp, Jumièges et Troarn ${ }^{44}$. La présence d'un office original normand dédié à saint Bénigne à Fécamp, au Mont-SaintMichel et à Jumièges, composé par Guillaume de Volpiano, a influencé la composition d'autres offices de saints normands où l'on retrouve des variantes des mêmes mélodies ${ }^{45}$. Ainsi l'office dédié à saint Taurin a été composé à Fécamp après la mort de Guillaume et est chanté à Fécamp et à Conches ${ }^{46}$. Si le chant grégorien importé par

38. Vers 1053-1055 Renouf, un moine du Mont, est fait abbé.

39. Conches : ORderic VITAL, Histoire ecclésiastique, II, p. 197. Troarn : ibid., II, p. 20 et 198.

40. ORDERIC VITAL, Histoire ecclésiastique, IV, p. 164.

41. DIARD, Olivier, Les offices propres dans le sanctoral normand, étude liturgique et musicale ( $X^{\mathrm{e}}$ $X V^{\mathrm{e}}$ siècles), thèse de doctorat, Université de Paris IV-Sorbonne, 2000, p. 106-108. Pour les solennités, ibid. Ouen, p. 130; Romain, p. 141; Philibert, p. 146-147 ; Aychadre, p. 150; Taurin, p. 165 et Leufroy, p. 170-171. J'adresse ici tous mes remerciements à Olivier Diard, dont les explications et les conseils relatifs à la liturgie et au chant m’ont été très précieux.

42. Quelques compositions de Guillaume de Volpiano, pour le cycle de Noël, dans LerouX, dom R., "Guillaume de Volpiano, son cursus liturgique au Mont-Saint-Michel et dans les abbayes normandes », dans Millénaire monastique du Mont-Saint-Michel, I, Histoire et vie monastique, dom Jean LAPORTE (éd.), Paris, Lethielleux, 1966, p. 442.

43. HeSBERT, dom René-Jean, Corpus antiphonalium officii 5, Fontes et earum prima ordinatio, Rome, Herder, 1975, p. 418 et 442.

44. Huglo, Michel, Les livres de chant liturgique, coll. Typologie des sources du Moyen Age occidental, fasc. 52, Turnhout, Brepols, 1988, p. 90.

45. Par exemple, pour l'office de saint Taurin : DiARD, Olivier, Les offices propres..., p. 232-236, 241, 243.

46. DiARD, Olivier, Les offices propres..., p. 165. Pour Fécamp : bréviaire de la Bibl. mun. de Rouen, ms 244 (A 261, $\mathrm{f}^{\circ} 253-256 \mathrm{v}^{\circ}$ ) ; 203 (Y 46), $\mathrm{f}^{\circ}$ 297-299; 251 (A 393, fo 152-156) et l'antiphonaire 245 (A 190), f $260 \mathrm{v}^{\circ}-263$. Pour Conches, mairie de Conches, Bréviaires : $\mathrm{ms} 3, \mathrm{f}^{\circ} 260 \mathrm{v}^{\circ}-262 \mathrm{v}^{\circ}$; $4, \mathrm{f}^{\circ} 182 \mathrm{v}^{\circ}-184$ et $\left.5, \mathrm{f}^{\circ} 206 \mathrm{v}^{\circ}-208\right)$ et le rituale, ms 6, $\mathrm{f}^{\circ} 120-120 \mathrm{v}^{\circ}$. 
Guillaume a effectivement remplacé le chant gallican, il lui a fallu imposer une théorie pour expliciter l'accord entre les mélodies permettant de réciter les psaumes (tons psalmodiques) et les chants les encadrant (antiennes) ${ }^{47}$. Conforme au tonaire de Saint-Bénigne de Dijon, le tonaire de Fécamp s'est répandu à Jumièges ${ }^{48}$.

Cinquièmement, l'influence clunisienne et dijonnaise est visible à Bernay, abbaye construite dans les années 1025-1030 et dont Guillaume de Volpiano a été l'abbé. Le plan échelonné à chevet, la présence de piles composées et celle d'une coursière au niveau supérieur du mur oriental du transept attestent l'influence bourguignonne ${ }^{49}$. En matière de décoration, le traitement et la manière d'organiser la figure dans un cadre d'un chapiteau de Bernay, conservé dans la crypte, orné d'un personnage à casque feuillu, pourraient indiquer des similitudes avec l'atelier de Saint-Bénigne ${ }^{50}$.

Sixièmement, la lecture des documents nécrologiques est révélatrice du souvenir d'un abbé entretenu par les communautés monastiques normandes, l'inscription au nécrologe donnant droit à des prières spéciales au chapitre. Le nom de Guillaume de Volpiano est consigné dans le nécrologe de Jumièges et dans le martyrologe du Mont-Saint-Michel, où l'office de saint Bénigne est chanté ${ }^{51}$. Mais ce qui ne manque pas d'étonner, c'est l'absence du nom de Guillaume de Volpiano dans les autres nécrologes conservés, au Bec, à Lyre, à Lessay et au Tréport. Certes dans ces établissements l'influence de Guillaume de Volpiano est quasi nulle. Mais comment expliquer son absence dans la documentation de Saint-Evroult et de Sées?

Ces quelques exemples invitent à considérer l'autorité de Guillaume de Volpiano, une autorité indéniable, mais peut-être localisée, circonscrite à quelques établissements : Fécamp et Jumièges au diocèse de Rouen, Bernay et Saint-Evroult au diocèse de Lisieux — le premier abbé de Saint-Evroult, Thierry de Mathonville, fut au préalable prieur de Jumièges sous l'abbatiat de Thierry -, Saint-Martin de Sées au diocèse de Sées, Le Mont-Saint-Michel au diocèse d'Avranches, Troarn, au diocèse de Bayeux, enfin Conches et Saint-Taurin d'Evreux au diocèse d'Evreux. Dans ces établissements, on doit néanmoins admettre des nuances à son autorité, à son influence. Saint-Evroult restauré en 1050 fait venir son premier abbé de Jumièges, mais dote son abbaye des coutumes clunisiennes pas exactement semblables à celle de Fécamp. Et Ordéric Vital, parfaitement bien renseigné sur ce qui se passe à Saint-Evroult, son monastère, souligne que l'abbé, Thierry de Mathonville, connaît bien aussi les coutumes de Saint-Vanne de Verdun et celles

47. DIARD, Olivier, Les offices propres..., p. 264-265.

48. Huglo, Michel, Les Tonaires. Inventaires, analyse, comparaison, Paris, Société de musicologie, Heugel et Cie, 1971, p. 332, pour les manuscrits de Jumièges, Bibl. mun. de Rouen, 248 (A 339) et de Fécamp, 244 (A 261).

49. BAYLÉ, Maylis, L'architecture normande..., p. 16.

50. GRODECKI, Louis, «Les débuts de la sculpture romane en Normandie. Les chapiteaux de Bernay ", Bulletin monumental, 1950, p. 33.

51. R.H.F., t. XXIII, p. 417. Bibl. Mun. Avranches, ms lat. 214, p. 6. 
que lui a enseignées Thierry de Jumièges ${ }^{52}$. Les premiers abbés de Conches et de Troarn sont des Fécampois qui laisseront rapidement leur place à des abbés choisis ailleurs qu'à Fécamp. À Conches, c'est à Saint-Laumer de Blois, à Coulombs au diocèse de Chartres ou au Bec qu'on va chercher des successeurs ${ }^{53}$. Les moines de Saint-Taurin d'Evreux, en 1104, se révoltent contre la tutelle fécampoise et l'abbé de Fécamp doit faire intervenir le duc-roi Henri Ier, en 1106, pour faire cesser ce qu'on est en droit d'appeler un mouvement de sécession ${ }^{54}$. À Bernay, dans la deuxième moitié du XII ${ }^{\mathrm{e}}$ siècle, les moines se tournent vers le Bec et en font venir deux abbés successifs ${ }^{55}$.

Examinons également les cas de trois abbayes qui ont au cours des $\mathrm{XI}^{\mathrm{e}}$ XII ${ }^{\mathrm{e}}$ siècles choisi un abbé fécampois sans que l'influence de Fécamp ait laissé des traces durables. Lorsque Nicolas, jeune moine de Fécamp et fils du duc Richard III, en 1042, prend la tête de Saint-Ouen de Rouen, il n'y impose pas l'office de Fécamp, bien au contraire ${ }^{56}$. En 1063, le duc Guillaume a choisi pour abbé de SaintWandrille, le moine de Fécamp, Gerbert. Ce dernier avait commencé par mener plusieurs expériences monastiques y compris en Italie avant son entrée à Fécamp en 1055. La situation chaotique qui règne à Saint-Wandrille après la mort de Gerbert en 1089, pendant deux ans, peut laisser supposer qu'il a peut-être cherché à bouleverser les coutumes en imposant celles de Fécamp. Par la suite, la sérénité revient avec le choix d'un abbé issu de la communauté ${ }^{57}$. L'abbaye de Notre-Dame d'Ivry, placée dès sa fondation en 1071 sous la tutelle de Notre-Dame de Coulombs, a un abbé fécampois au XII ${ }^{e}$ siècle, dans des circonstances obscures ${ }^{58}$.

Il est plusieurs établissements où l'autorité de Guillaume n'a pas droit de cité. Dans les années 1030, c'est-à-dire au lendemain de la mort de Guillaume de Volpiano, plusieurs abbayes nouvellement fondées tournent indéniablement le dos à Fécamp. Ainsi, à La Trinité du-Mont de Rouen, le premier abbé Isembert, qui fut moine de Saint-Ouen, apporte avec lui l'office de saint Ouen et celui de saint Nicolas, qu'il impose ${ }^{59}$. Son successeur choisit les abbés de Cormeilles et du Tréport. Son disciple, Ainard, est installé à Saint-Pierre-sur-Dives, au diocèse de Sées, abbaye fondée par les comtes d'Eu, futurs fondateurs du Tréport ${ }^{60}$. En 1032, le premier abbé de Cerisy vient aussi de Saint-Ouen ${ }^{61}$. La vita d'Herluin, le fondateur et premier abbé du Bec, rapporte que ce dernier, en quête d'un monastère après

52. Histoire ecclésiastique, II, p. 74.

53. Robert De Torigni, De immutatione..., II, p. 198.

54. Gallia christiana, t. XI, instrumenta, col. 127-128.

55. Gallia christiana, t. XI, col. 832 A.

56. L'enquête du Corpus antiphonalium officii, op. cit., montre un cursus liturgique indépendant de celui de Fécamp. Diard, Olivier, op. cit., p. 274 et l'analyse du sanctoral, p. 617.

57. LAPORTE, Dom Jean, " La fin du XI ${ }^{\mathrm{e}}$ siècle et le début du XII ${ }^{\mathrm{e}}$ siècle ", L'abbaye de Saint-Wandrille de Fontenelle, $\mathrm{n}^{\circ} 29,1980$, p. 23.

58. Gallia christiana, t. XI, col. 653 A

59. DIARD, Olivier, Les offices propres..., p. 271, 274 et 514.

60. ORDERIC VITAL, II, Histoire ecclésiastique, p. 352.

61. Robert de TORIGNi, De immutatione..., II, p. 195. 
l'abandon de la vie laïque, s'est arrêté au début des années 1030 à Bernay, où il fut rudoyé par le custos, Raoul, un moine de Fécamp ${ }^{62}$. Herluin fonde finalement un monastère au Bec en 1034, dans la vallée de la Risle ${ }^{63}$. L'Italien Lanfranc fréquente les écoles d'Avranches au plus tard en 1039 et s'arrête au Bec $^{64}$. Le nombre d'abbés, formés au Bec et envoyés aux quatre coins de la Normandie, puis de l'Angleterre après 1066, est largement supérieur à celui des Fécampois ${ }^{65}$.

Il convient dès lors de se poser la question de savoir si Guillaume de Volpiano lui-même n'a pas entrevu les limites de son action avant même son arrivée. En effet, on s'explique difficilement pourquoi Maieul de Cluny dans un premier temps, puis Guillaume de Volpiano ont commencé par refuser de répondre à l'appel des ducs à venir réformer le monachisme normand. Le Libellus de revelatione, composé sous l'abbatiat de Guillaume de Rots (1079-1107), dans le souci de consacrer le caractère singulier et exceptionnel de l'abbaye et dont Mathieu Arnoux a montré le crédit qu'on doit apporter à ses informations, indique que Guillaume de Volpiano redoute les Normands, « des hommes barbares et farouches et démolisseurs des temples saints ${ }^{66}$. Et pourtant, les travaux récents de Katharine KeatsRohan mettent l'accent sur une donation de terre au Mont-Saint-Michel, en Touraine, effectuée par Maieul de Cluny le 21 mars $966^{67}$. Certes, cela démontre l'intérêt porté au monachisme de la principauté normande par Cluny, mais à cette époque l'abbaye du Mont-Saint-Michel demeure encore dans l'orbite des régions situées à l'ouest de la principauté. Et Maieul, qui connaît l'Eglise normande, mais au prisme du Mont-Saint-Michel, n'ose pas encore à la fin du X'e siècle, après la révolte paysanne de 996 , lancer ses troupes dans la partie orientale du duché, ce qui explique l'accusation lancée aux légats envoyés par le duc à Guillaume de Volpiano. Maieul n'a pas souhaité disperser ses efforts sous le règne de Richard Ier et au début de celui de Richard II. L'hésitation n'est plus de mise à la toute fin du $\mathrm{X}^{\mathrm{e}}$ siècle. Une donation de Richard I ${ }^{e r}$ à l'abbaye de Saint-Bénigne de Dijon montre que même si les parties concernées avaient préparé le terrain de la venue des Clunisiens, le moment n'est propice qu'en $1001^{68}$. Les dernières hésitations sont levées par l'obtention du silence de l'archevêque Robert, qu'on n'entend pas et qui ne souscrit pas l'acte de 1006. Il faut attendre 1023 pour qu'apparaissent conjoin-

62. Vita domni Herluini abbatis Beccensis, J. ARMITAGE RoBInSON (éd.), Gilbert Crispin abbot of Westminster, A study of the abbey under Norman Rule, Cambridge, 1911, p. 92.

63. FAUROUX, Recueil..., nº 4, p. 31.

64. GIBSON, Margaret, Lanfranc of Bec, Oxford, 1978, p. 15.

65. Voir notre étude en préparation sur les abbés bénédictins en Normandie à l'époque ducale.

66. ARNOUX, Mathieu, «La fortune du Libellus de revelatione, edificatione et auctoritate Fiscannensis monasterii. Note sur la production historiographique d'une abbaye bénédictine normande », Revue d'histoire des textes, t. XXI, 1991, p. 135-158.

67. Keats-Rohan, Katharine, «Une charte de l'abbé Mayeul de Cluny et la réforme du Mont-SaintMichel, annexe ", La Normandie vers l'an Mil, François de BEAUREPAIRE et Jean-Pierre CHALINE (coord.), Société de l'Histoire de Normandie, Rouen, 2000, p. 166-167.

68. FAUROUX, Recueil..., n 17, p. 23. 
tement dans un acte ducal en faveur de Fécamp les attestations de l'archevêque Robert et celle de l'abbé Guillaume ${ }^{69}$.

En réalité, Guillaume de Volpiano n'arrive pas en terrain vierge. D’une part, le monachisme n'a jamais totalement disparu en Normandie, d'autre part, bien avant l'arrivée de Guillaume, des réformateurs sont à pied d'œuvre. Un très rapide tour d'horizon à partir des derniers travaux de Jacques Le Maho, Katharine KeatsRohan, Mathieu Arnoux et des nôtres permet de tenir pour une hypothèse solide, sinon d'affirmer que des moines n'ont cessé d'être présents au Mont-Saint-Michel, que des clercs, voire des moines, ont dû continuer de mener une vie communautaire à Saint-Evroult ainsi qu'à La Croix-Saint-Leufroy. Jacques Le Maho, par une enquête d'une grande minutie, parvient à vider de son sens la théorie de la disparition du monachisme dans la vallée de la Seine en pleine période d'invasion viking. On sait maintenant qu'à la fin du IX ${ }^{\mathrm{e}}$ siècle, des communautés exilées depuis le Cotentin, ont trouvé refuge à plusieurs endroits dans cette vallée, en tout état de cause à Fécamp et près de Jumièges ${ }^{70}$. À Saint-Ouen de Rouen, tout laisse à penser que sous Rollon les moines sont revenus. Au début du $\mathrm{X}^{\mathrm{e}}$ siècle, l'archevêque exerce encore conjointement sa charge avec celle d'abbé, comme aux temps mérovingiens et carolingiens. Un abbé régulier, Hildebert, est présent en $989 \mathrm{au}$ moment de la translation des reliques de saint Ouen et peut-être tient-il les rênes de l'abbaye dès $960^{71}$. À Jumièges, les efforts de Guillaume Longue Epée pour ranimer la vie monastique en faisant venir l'abbé Martin et des moines de Saint-Cyprien de Poitiers, que méconnaît le dernier ouvrage de l'historienne américaine, Cassandra Potts consacré au monachisme en Normandie, ne sont absolument pas sans lendemain après l'assassinat du duc le 17 décembre $942^{72}$. Un scriptorium et un atelier d'enluminures y existent ${ }^{73}$. À Saint-Wandrille dès 959, Mainard le disciple de Gérard de Brogne, grand réformateur d'abbayes en Flandre, fait renaître le monachisme. En réalité, la communauté expatriée au temps des Vikings n'a pas totalement disparu et si l'on admet aujourd'hui que le Mainard de Saint-Wandrille n'est jamais parti en 966 au Mont-Saint-Michel, il a pu rester longtemps dans l'abbaye renaissante et y instaurer une communauté forte. En 1001, c'est vraisemblablement un des trois rectores, connus seulement par le Gallia christiana

69. Fauroux, Recueil..., $\mathrm{n}^{\circ} 25$.

70. LE MAHO, Jacques, «Un exode de reliques dans les pays de la Basse Seine à la fin du IX siècle », Bulletin de la Commission départementale des Antiquités de la Seine-Maritime, t. XLVI, 1998, p. 136-188.

71. Il est présent à la seconde translation de saint Ouen en 989 (Patrologie latine, t. CLXII, col. 1162). Le Neustria pia, p. 21 s'appuyant sur un Vetus chronicon, indique une durée d'abbatiat de 46 ans.

72. Pоттs, Cassandra, Monastic Revival and Regional Identity in Early Normandy, Studies in the History of Medieval Religion, XI, Woodbridge, The Boydell Press, 1997.

73. NORTIER-MARCHAND, Geneviève, "La bibliothèque de Jumièges au Moyen Age ", in Jumièges, congrès scientifique du XIIIe centenaire, Rouen, 10-12 juin 1954, Rouen, 1955, II, p. 601. HowE, John, "The Hagiography of Jumièges (Province of Haute Normandie) », in L'hagiographie du Haut Moyen Age en Gaule du Nord, manuscrits, textes et centres de production, Beihefte der Francia, 52, 2001, p. 96-97. 
qui ne cite pas ses sources, qui est à la tête de Saint-Wandrille. Le premier des trois rectores Enfulbert, est mort en 993 après avoir "restauré les basiliques», sans doute les églises antérieures aux Vikings. Il eut même le droit à une épitaphe. Ces rectores ne pourraient-ils pas être des sortes de custos avant la lettre? Enfulbert, enterré à Jumièges, dont il avait été d'abord moine et doyen, aurait pu être custos de l'abbaye de Saint-Wandrille placée momentanément sous la férule de Jumièges. Cela donnerait consistance à l'hypothèse de la stabilité du monachisme gémmetique à la fin du Xe siècle, dont on connaît certes mais assez mal les abbés. En 1008, alors que Guillaume de Volpiano est à Fécamp, c'est Gérard, un abbé de Crépyen-Valois, qui accède à l'abbatiat de Saint-Wandrille ${ }^{74}$. A priori, les liens avec Jumièges, s'ils ont existé, sont rompus et l'on sait que Saint-Wandrille fera cavalier seul. Mais Gérard est inscrit au 29 novembre dans le nécrologe et le martyrologe $\mathrm{du}$ Mont-Saint-Michel ${ }^{75}$. L'insistance avec laquelle Dom Pommeraye au XVII siècle a voulu absolument que les abbés rouennais du début du XIe siècle aient été mis en place par Guillaume de Volpiano, n'a pas de fondement. Certes, l'origine des abbés Henri et Herfast de Saint-Ouen risque de demeurer à jamais mystérieuse, mais rien ne peut prouver qu'ils viennent de Fécamp. Au MontSaint-Michel, la communauté est bien solide en 1001 sous la férule de l'abbé Mainard II, qui gouverne depuis 991. À Fécamp même, une lecture attentive du Libellus permet de saisir qu'entre l'installation en 990 des chanoines par Richard I ${ }^{\text {er }}$ et l'arrivée de Guillaume de Volpiano, des moines ont occupé les bâtiments construits par Richard $\mathrm{II}^{76}$.

Le monachisme n'est pas mort des invasions vikings. À cet égard, les travaux de l'Américaine, Felice Lifshitz, s'ils doivent être lus avec prudence, ont contribué à mettre en évidence la responsabilité des troubles des guerres internes en Neustrie au $\mathrm{X}^{\mathrm{e}}$ siècle, dans le déclin du monachisme ${ }^{77}$. Guillaume n'a donc pas eu à relever de ses cendres le monachisme en Normandie. Le renouveau monastique dans la nouvelle principauté est multiforme, entre les mains de réformateurs nombreux, issus de régions diverses extérieures au duché. Loin d'être un espace clos, le duché est une principauté importante de l'Occident chrétien et à ce titre reçoit les influences variées. Il prend appui sur des communautés qui ne sont pas disparues, loin s'en faut.

$\mathrm{Au}$ terme de ce rapide tour d'horizon, il faudra s'interroger sur les moments historiographiques, postérieurs au Moyen Age, qui ont porté au pinacle Guillaume de Volpiano. Dom Pommeraye au XVII siècle voit Guillaume à l'œu-

74. Inventio et miracula sancti Vulfranni, Jean LAPORTE (éd.), Société de l'Histoire de Normandie, Mélanges, 14 série, Rouen-Paris, 1938, \$30, p. 43.

75. Bibl. Mun. Avranches, ms 214, p. 180. LAPORTE, Jean, «Les obituaires du Mont-Saint-Michel», Millénaire monastique du Mont-Saint-Michel, I, p. 738.

76. Patrologie latine, t. CLI, col. 720.

77. Par exemple : LIFSHITZ, Felice, «The « exodus of holy bodies » reconsidered : the translation of the relics of St. Gildard of Rouen to Soissons ", Analecta Bollandiana, t. 110, fasc. 3-4, 1992, p. 329-340. 
vre dans de nombreux monastères ${ }^{78}$. Certes, on l'a montré, Guillaume place ses disciples à la tête de plusieurs monastères. Mais, lorsqu'il arrive en Normandie, d'autres réformateurs sont déjà à l'œuvre. Plus près de nous, Jean-François Lemarignier considérait comme sans avenir les efforts de Martin de Poitiers à Jumièges $^{79}$ ! Laissons le dernier mot à trois grands de la littérature médiévale normande. Guillaume de Poitiers et Guillaume de Jumièges ne prennent pas la peine de mentionner Guillaume de Volpiano. Quant à Ordéric Vital, il ne lui accorde en tout et pour tout que deux mentions assez laconiques, la première faisant de lui un homme sage et très fervent, "vir sapiens et in religione ferventissimus ", la seconde indiquant qu'il a édifié le monastère « sollerter et religiose ${ }^{80}$. Ces formules n'ont rien à voir avec les envolées oratoires consacrées à d'autres abbés par le moine d'Ouche! Peut-on se résigner à dire qu'elles sont simplement à la mesure de l'œuvre de Guillaume de Volpiano ? Certainement pas car Ordéric jalouse sans doute quelque peu le monastère fécampois. Oeuvre exagérée ensuite par une certaine historiographie ? Certainement. Nous sommes tentés de revenir sur l'hésitation première de Guillaume de Volpiano, auquel Hugues de Flavigny dans sa Chronique écrite avant la fin du XIe siècle, donne une réputation de rigueur, le surnommant "super-règle ${ }^{81}$. Même son biographe Raoul Glaber au chapitre V du Troisième Livre des Histoires met l'accent sur les calomnies suscitées par l'observance de la Règle ${ }^{82}$. N'avait-il pas pressenti l'ingratitude de la tâche ? Quoiqu'il en soit, l'œuvre de Guillaume de Volpiano suscite des questionnements sur la diversité du monachisme, auquel la principauté puise singulièrement sa force.

78. Dom Pommeraye s'interroge sur le rôle de l'abbé italo-dijonnais à propos d'un passage de la Chronique de l'abbaye de Saint-Bénigne de Dijon (p. 158-159) qui indique que Richard II a mis Guillaume de Volpiano à la tête de Jumièges, de Saint-Ouen et du Mont-Saint-Michel. (POMMERAYE, Jean-François, Histoire de l'abbaye royale de Saint-Ouen de Rouen, Rouen, 1662, p. $247-$ 248). Dom Pommeraye conclut que Guillaume a remis l'abbaye dans «l'étroite observance... sans avoir la qualité d'abbé de Saint-Ouen, non plus que de Jumièges, du Mont-Saint-Michel ».

79. "Jumièges et le monachisme occidental au haut Moyen Age (VII ${ }^{\mathrm{e}}-\mathrm{XI}{ }^{\mathrm{e}}$ siècle). Quelques observations ", Jumièges, congrès du XIII centenaire, II, p. 762.

80. ORDERIC VITAL, Histoire ecclésiastique, II, p. 292 et VI, p. 40.

81. Hugues de FlavignY, Chronicon Hugonis monachi Virdunensis et Divionensis, abbatis Flaviniacensis, PerTz (éd.), M.G.H., S.S., t. VIII, 1848, p. 391, 31-32.

82. Histoires, ARNouX, Mathieu (éd.), Turnhout, Brepols, 1996, p. 171, § 16. 